\title{
Proofreading Tile Sets: Error Correction for Algorithmic Self-Assembly
}

\author{
Erik Winfree and Renat Bekbolatov \\ Computer Science and Computation \& Neural Systems \\ California Institute of Technology, Pasadena, CA 911125, USA
}

\begin{abstract}
For robust molecular implementation of tile-based algorithmic self-assembly, methods for reducing errors must be developed. Previous studies suggested that by control of physical conditions, such as temperature and the concentration of tiles, errors $(\varepsilon)$ can be reduced to an arbitrarily low rate - but at the cost of reduced speed $(r)$ for the self-assembly process. For tile sets directly implementing blocked cellular automata, it was shown that $r \approx \beta \varepsilon^{2}$ was optimal. Here, we show that an improved construction, which we refer to as proofreading tile sets, can in principle exploit the cooperativity of tile assembly reactions to dramatically improve the scaling behavior to $r \approx \beta \varepsilon$ and better. This suggests that existing DNA-based molecular tile approaches may be improved to produce macroscopic algorithmic crystals with few errors. Generalizations and limitations of the proofreading tile set construction are discussed.
\end{abstract}

\section{Introduction}

The experimental demonstration that DNA can be used to encode and process information [1] has stimulated interest in how biomolecular processes can be programmed to carry out logical algorithms. Algorithmic self-assembly of DNA tiles has been proposed as the basis for parallel computation of solutions to hard combinatorial problems $[27,30,18,12]$ and for bottom-up nanofabrication of complex structures that can be specified by simple rules $[23,20,8]$.

Understanding of this approach relies upon an abstract model of the growth process, known as the abstract Tile Assembly Model (aTAM). As in Wang's Tiling Problem $[25,26]$, a tile system consists of a finite set of square tiles with a label on each side. For simplicity, tiles cannot be rotated. The aTAM augments these tiles with a strength function that specifies how tightly tiles stick to each other when the labels on touching sides match (referred to as a bond). This motivates a growth rule: starting with a specified seed tile, a new tile may be added at any position where the total strength of all newly-formed bonds exceeds a threshold, $\tau$. This rule is intrinsically asynchronous and non-deterministic: only certain tile sets will produce a uniquely-defined structure. The aTAM is illustrated in figure 1ab, using a tile set we call the Sierpinski tiles; at $\tau=2$, the growth results [28] in an infinite Sierpinski triangle pattern [5]. 

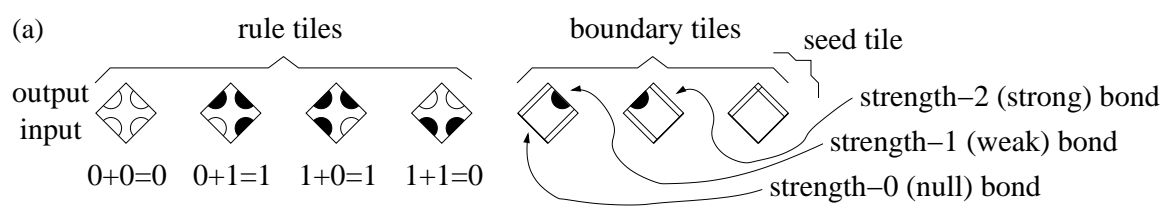

(b)
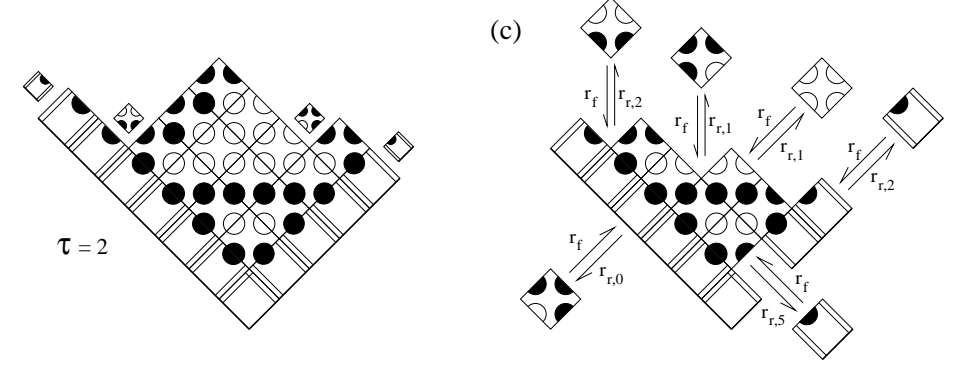

Fig. 1. (a) The seven Sierpinski tiles include four rule tiles implementing the XOR logic and three boundary tiles, including the corner tile which is used as the seed tile. Whereas all sides of the rule tiles form strength-1 (weak) bonds, the boundary tiles also make use of strength-2 (strong) bonds and strength-0 (null) bonds. Strong bonds are drawn as double lines, and null bonds are drawn as thick lines. This construction can be trivially generalized to implement an arbitrary BCA with more than two symbols. (b) Growth of the Sierpinski tiles from the seed tile according to the aTAM at $\tau=2$. The small tiles indicate the (only) four sites where growth can occur. At each location, there is a unique tile that may be added, and a unique pattern results. (c) Rates for tile addition and tile dissociation in the kTAM: $r_{f}$ is the forward rate for association of any tile at any site, and $r_{r, b}$ is the reverse rate for dissociation of a tile that makes bonds with total strength $b$. All and only single monomer tile associations and dissociation events are considered in the kTAM; a representative selection is shown here.

The Sierpinski rule tiles implement a replacement rule $(x, y) \rightarrow(z, z)$ where $x, y \in\{0,1\}$ and $z=(x+y) \bmod 2$; each tile has some $x$ and some $y$ on the bottom two sides and the corresponding $z$ on both top sides. This is an instance of a one-dimensional blocked cellular automaton (BCA): an initial string, the bottom layer, is transformed for each subsequent layer by partitioning the string into pairs (alternately odd/even-indexed and even/odd-indexed elements) and replacing each pair according to a rule $(x, y) \rightarrow\left(f_{1}(x, y), f_{2}(x, y)\right)$, where all values are in an alphabet, e.g., $\{0,1, \ldots, N\}$. Generalizing the Sierpinski rule tiles to rule tiles whose inputs and outputs may take on $(N+1)$ values yields a direct implementation for any chosen BCA: one rule tile is used for each of $(N+1)^{2}$ possible input pairs, and the initial conditions for the computation are given using boundary tiles with strength-2 bonds that grow from the seed tile. We call this the direct tile set for a given BCA. Since BCA are capable of Turing-universal computation, this implies that tile-based self-assembly provides a natural logical basis for programmable self-assembly processes with arbitrary complexity. We now need to find a physical process, such as DNA tile assembly, wherein self-assembly occurs (at least approximately) according to the aTAM rules at $\tau=2$, using tiles with bond strengths restricted to 0,1 , and 2 . 
Tile-based self-assembly of two-dimensional periodic structures has been successfully demonstrated experimentally using a variety of molecular implementations of DNA tiles $[29,11,14,31]$. The key principle is that each DNA molecule has four short single-stranded regions, known as sticky ends, which direct how the DNA tiles bind to each other: sticky ends with complementary sequences can form a thermodynamically favorable double-helix, as shown in figure 2 for DNA tiles made from DNA double-crossover molecules [9]. Attempts at algorithmic self-assembly in one dimension [13] and in two dimensions [19] have also proven successful, but with two limitations: (1) incorrect tiles are incorporated into the growing structure with error rates ranging from $1 \%$ to $10 \%$, and (2) spurious nucleation (not involving the seed tile) results in many structures that perform the wrong computation and thus produce an undesired structure.

Our concern in this paper is how to control growth errors. ${ }^{1}$ We can consider four approaches to reducing growth errors.

Logical error correction. Accept an intrinsic error rate $\varepsilon$, and design a larger tile set that contains logic to detect and correct errors. In principle, this should be possible by making use of one-dimensional fault-tolerant cellular automata [10], but it is likely to be extremely complicated.

Optimized physical conditions. Study how physical conditions, such as temperature, tile concentrations, and buffer conditions, determine the error rate, and optimize them to obtain the best performance. As was shown in [28], this approach is promising, but it is likely to require extremely slow growth conditions.

New molecular mechanisms. Devise new physical mechanisms, such as, for example, more complicated molecular implementation of tiles with latches and switches. However, new structural motifs are difficult to design and characterize, so this approach must be considered with caution.

Exploiting cooperative binding. While retaining the original molecular tile design, redesign the original tile set to exploit physical mechanisms already inherent in the self-assembly process. This combined approach, which relies both on logical aspects of the tile set and on physical aspects of the assembly process, is explored here with dramatic benefits.

\section{The kinetic Tile Assembly Model: error vs growth rates}

Both growth errors and nucleation errors can be understood in terms of an extension of the aTAM to include rates both for tiles associating to and for tiles dissociating from the growing crystal (figure 1c); this model is known as the kinetic Tile Assembly Model (kTAM) [28]. The on-rates and off-rates can be chosen according to the principles of DNA hybridization [4], as illustrated in figure 2 for tiles implemented as DNA double-crossover (DX) molecules [9]. The fundamental observation is that while on-rates depend only upon the concentration of the tiles, the off-rates depend exponentially upon the total strength of

\footnotetext{
${ }^{1}$ Nucleation errors will be treated in an upcoming paper [22].
} 
(a)
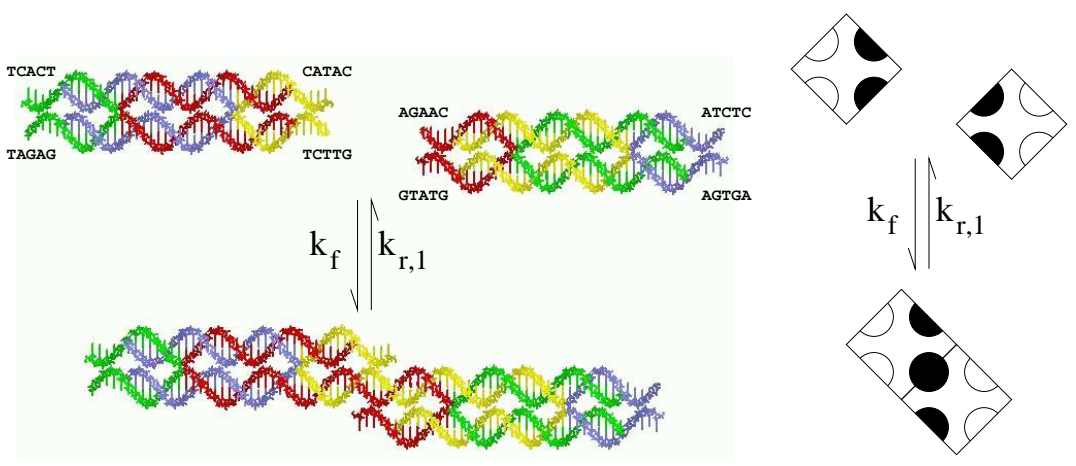

(b)
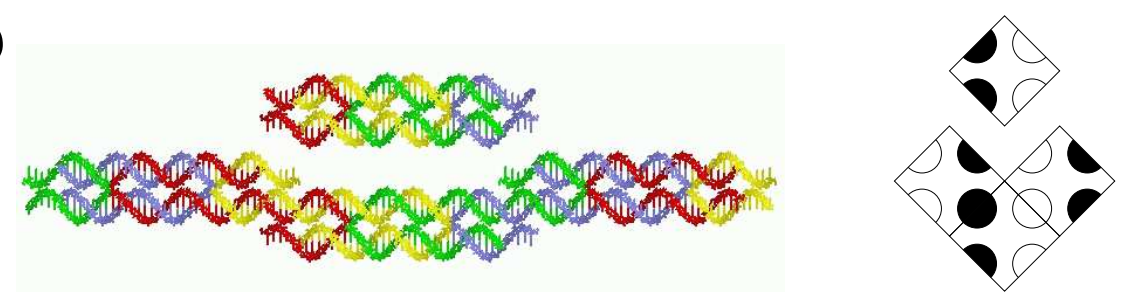

$$
\mathrm{k}_{\mathrm{f}} \| \mathrm{k}_{\mathrm{r}, 2}
$$
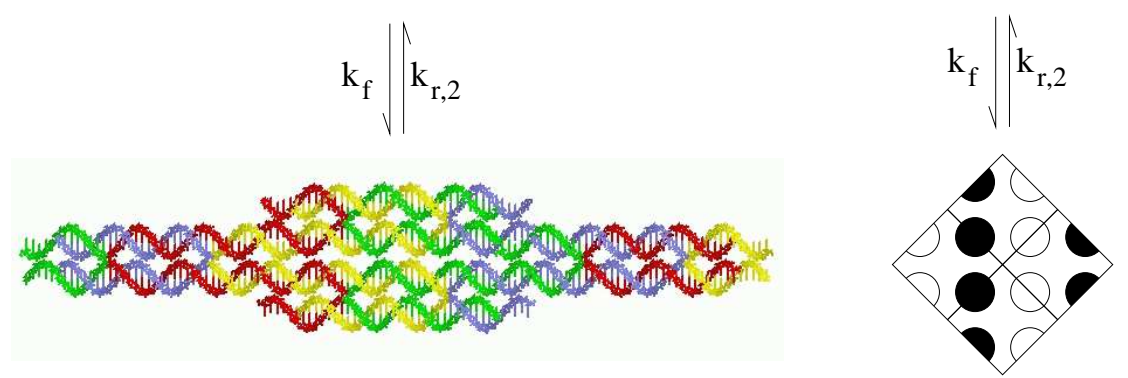

Fig. 2. (a) Assembly of two double-crossover tiles via hybridization of 5-nucleotide sticky ends. $k_{f}$ is the forward rate constant, in $/ M / s e c$, and $k_{r, 1}=k_{f} e^{-G_{s e}}$ is the reverse rate constant, in / sec. (b) Assembly of a double-crossover tile into a site on the growth front of a crystal via hybridization of two 5 -nucleotide sticky-end pairs. The forward rate constant is assumed to be the same as for the single sticky-end reaction of (a), while the reverse rate constant is assumed to require twice as much energy to simultaneously break both sticky-end bonds - i.e., binding is cooperative - and thus $k_{r, 2}=k_{f} e^{-2 G_{s e}} . G_{s e}$ is the free energy of dissociation for a single sticky end, in units of $R T$.

molecular interactions, i.e., the number of base pairs that must be broken in order for the tile to dissociate. Thus, single tiles (monomers) that either totally or partially mismatch their neighbors arrive at a site with equal frequency as tiles that correctly match their neighbors, but the correctly-matching tiles stay much 
longer. These considerations suggest that behavior of the system is characterized by two essential physical parameters, $G_{m c}$ and $G_{s e}$, respectively measuring the monomer concentration and the sticky-end bond strength as unitless free energies. Specifically, we define [monomer tile] $/ M=e^{-G_{m c}}$, and thus $G_{m c}$ is primarily entropic, as it measures the spatial degrees of freedom that are lost when a free-floating monomer tile is localized on the assembly. ${ }^{2}$ Similarly, we define the free energy of dissociation of a single sticky end to be $\Delta G / R T=G_{s e}$, and thus $G_{s e}$ contains a mix of entropic and enthalpic factors related to the formation of the double-helix, measured in units of $R T$.

We can now formulate the kTAM to describe the growth of a single crystal in an environment where the concentration of monomer tiles remains fixed. Absolute rates for events affecting this crystal are given by

$$
r_{f}=k_{f}[\text { monomer tile }]=k_{f} e^{-G_{m c}}
$$

for association of a new monomer tile at any given site, and

$$
r_{r, b}=k_{r, b}=k_{f} e^{-b G_{s e}}
$$

for dissociation of a tile whose interactions with the crystal sum to $b$, in the "strength units" of the aTAM. $b$ can be thought of as the effective number of unit-strength sticky ends binding the tile to the crystal. These rates specify a continuous-time Markov process (satisfying detailed balance) for modeling the growth of a single crystal in a solution of free monomer tiles. ${ }^{3}$

\footnotetext{
${ }^{2}$ The simplest situation is when all monomer tile species are present at the same concentration of each species. In some cases, it is convenient to specify the stoichiometry of the tile species, relative to the "generic" monomer tile whose concentration is determined directly by $G_{m c}$. For example, in the Sierpinski tile simulations, the boundary tiles are present at half the concentration of the rule tiles, which ensures that the boundary grows at approximately the same speed as the interior.

${ }^{3}$ Our model assumes that only single tiles associate to and dissociate from an assembly. Solution will contain a distribution of assembly types, from dimers (two tiles bound to each other) on up. However, near the melting temperature for crystals, where our results hold, dimer concentrations should be significantly lower than monomer concentrations, and thus dimer association events should be rare. Likewise, a pair of connected tiles may simultaneously dissociate from an assembly, or assemblies can even fracture into two or more large pieces. However, the energy required for such events is typically significantly greater than that for monomer dissociation. Therefore, we do not expect our results to change qualitatively if evaluated under more sophisticated models. For the tile sets discussed here, we have seen only minor quantitative changes when either (a) the model also allows tile dissociation events wherein after removal of the tile, the assembly falls apart into two unconnected pieces (such reactions are not reversible in the context of a single-crystal model, and thus are excluded from the standard kTAM), or (b) additionally, the model allows dimers or $2 \times 2$ blocks to dissociate together. There are tile sets for which these modification do have qualitatively significant ramifications, for example, tile sets involving linear polymerization or blocks of tiles that are strongly bound to each other but have weak interactions with the crystal.
} 


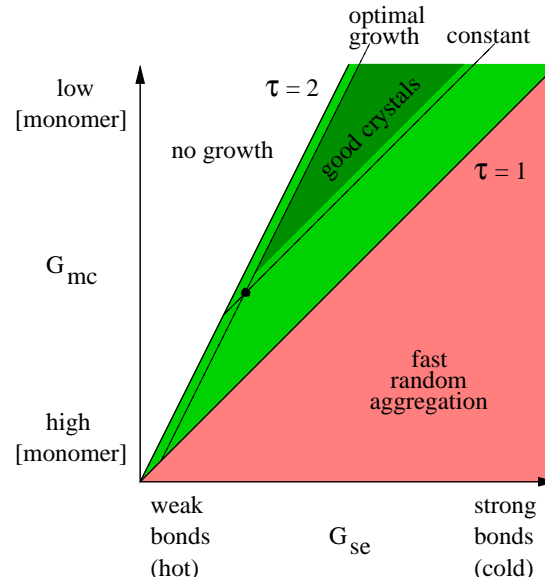

(a)
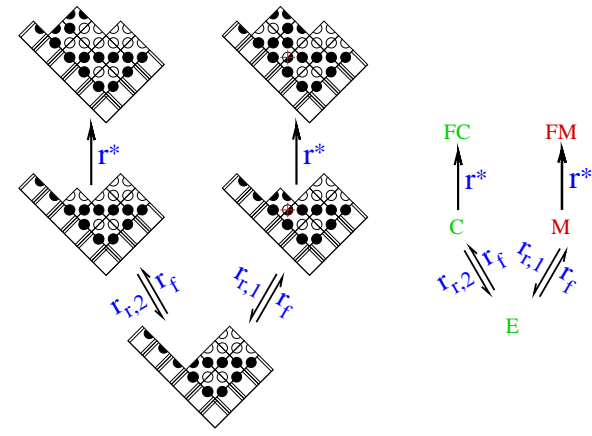

(b)

Fig. 3. (a) Phase diagram [28] for crystal growth of tiles implementing a BCA, under the kTAM. "Good crystals" (growth rate comparable to $k_{f}[D X]$ and error rate smaller than $\varepsilon$ ) are obtained for large $G_{s e}$ and $G_{m c}$, below the $\tau=2$ boundary marking the melting transition where $G_{m c}=2 G_{s e}$. (b) Model for kinetic trapping. The growth site may $(E)$ be empty; $(C)$ contain a correct tile; $(M)$ contain a mismatched tile; $(F C)$ be "frozen" with the correct tile in place; or $(F M)$ be "frozen" with the mismatched tile. $r^{*}$ represents the rate at which tiles on the growth front are covered. The error rate is taken to be the probability that, starting in $E$, the system reaches $F M$.

The parameters $G_{m c}$ and $G_{s e}$ represent the "physical conditions" under which tile-based assembly can take place. $G_{m c}$ can be made large (or small) by using DNA tiles at low (or high) concentrations. $G_{s e}$ can be made large (or small) by letting the self-assembly take place at a cold (or hot) temperature. ${ }^{4}$ For what settings of these parameters does the kTAM obey the aTAM rules with high probability? First note that if $2 G_{s e}>G_{m c}>G_{s e}$, then the tile additions shown in figure $1 \mathrm{~b}$ are favorable, as $r_{f}>r_{r, 2}$, but all other tile additions are unfavorable, as they make at most 1 bond and $r_{f}<r_{r, 1}$. Thus, the aTAM correctly abstracts which reactions are favorable, and which are unfavorable, with respect to the kTAM. However, in the kTAM, unfavorable reactions also occur with some frequency, so we expect assembly errors. Figure 4a shows several snapshots from a Monte Carlo stochastic simulation; single growth errors occur in the $3^{\text {rd }}$ and $4^{\text {th }}$ frames, causing subsequent error-free growth to develop into an undesired pattern. How frequent are these errors, and how can they be minimized?

\footnotetext{
${ }^{4}$ Naturally, the assumption that $G_{m c}$ and $G_{s e}$ both remain constant is likely to be violated in actual experiments, both for reasons under our control (e.g., using a temperature annealing schedule) and for reasons not easily under our control (e.g., the depletion of ambient monomer tile concentrations as a significant fraction of tiles become incorporated into crystal assemblies.
} 
Previous studies [28] using this model showed that both growth errors and certain nucleation errors ${ }^{5}$ can be controlled in the limit of low monomer tile concentrations (i.e., large $G_{m c}$ ) and strong sticky-end interactions (i.e., large $G_{s e}$ ) so long as the system is kept near the melting temperature of the crystal (i.e., $G_{m c} \approx 2 G_{s e}$ ). As shown in figure $3 \mathrm{a}$, phase space can be divided into three regions: no crystal growth occurs if $G_{m c}>2 G_{s e}$; algorithmic self-assembly (with some error rate) occurs for $2 G_{s e}>G_{m c}>G_{s e}$; and essentially random aggregation is obtained for $G_{s e}>G_{m c}$. Within the algorithmic phase, two factors limit the performance that can be achieved: thermodynamics tells us the best error rate that can be achieved at equilibrium given the energetics of the system, while the kinetics determine how quickly we get there - if at all.

If self-assembly achieves equilibrium, the probability of observing a particular assembly $A$ will be governed by the Boltzman equation:

$$
\operatorname{Pr}(A)=\frac{1}{Z} e^{-G(A)} \quad \text { with } \quad Z=\sum_{A^{\prime}} e^{-G\left(A^{\prime}\right)}
$$

where $G(A)=n G_{m c}-b G_{s e}$ is the free energy of the assembly, $n$ is the number of tiles in the assembly, $b$ is the total of all bond strengths in the assembly, and $Z$ is the partition function. Thus, an $n$-tile assembly that has $\Delta b$ more bond strength than another $n$-tile assembly will be $e^{\Delta b G_{s e}}$ more likely. In a direct BCA tile set with $(N+1)$ states, a typical growth site will present two sides with strength-1 bonds, a unique correct tile will match both bonds, and there will be exactly $N$ competing tiles that have a mismatch on the left side, as well as $N$ that have a mismatch on the right side. It follows that the equilibrium probability of incorporating the correct tile at a given growth site is

$$
1-\varepsilon \approx \frac{e^{2 G_{s e}}}{e^{2 G_{s e}}+2 N e^{G_{s e}}}, \text { and thus } \varepsilon \approx 2 N e^{-G_{s e}}
$$

where $\varepsilon$ is the per-tile error rate. It is mildly surprising that $G_{m c}$ has no effect on the equilibrium error rates.

However, due to kinetics, equilibrium is seldom achieved far below the melting transition. The primary cause of growth errors in this case was found to be a form of kinetic trapping, wherein tiles that associate with a mismatch on the growth front don't have time to dissociate, and are frozen in place by further growth; thus equilibrium error rates are observed only near the melting transition. The essential feature of kinetic trapping within BCA tile self-assembly is that once an error has occurred, both sites above the mismatched tile display an $(x, y)$ pair that is perfectly matched by some monomer tile in solution, because tiles implementing all replacement rules $(x, y) \rightarrow\left(f_{1}(x, y), f_{2}(x, y)\right)$ are present. Thus,

\footnotetext{
${ }^{5}$ It was shown that spurious nucleation of rule tiles can be controlled, for essentially the same reason that supersaturated solutions can be maintained: there is a critical nucleus size (based on surface-to-volume energies) beyond which growth is favorable and below which growth is unfavorable. However, spurious nucleation of boundaries is a more difficult issue [21].
} 


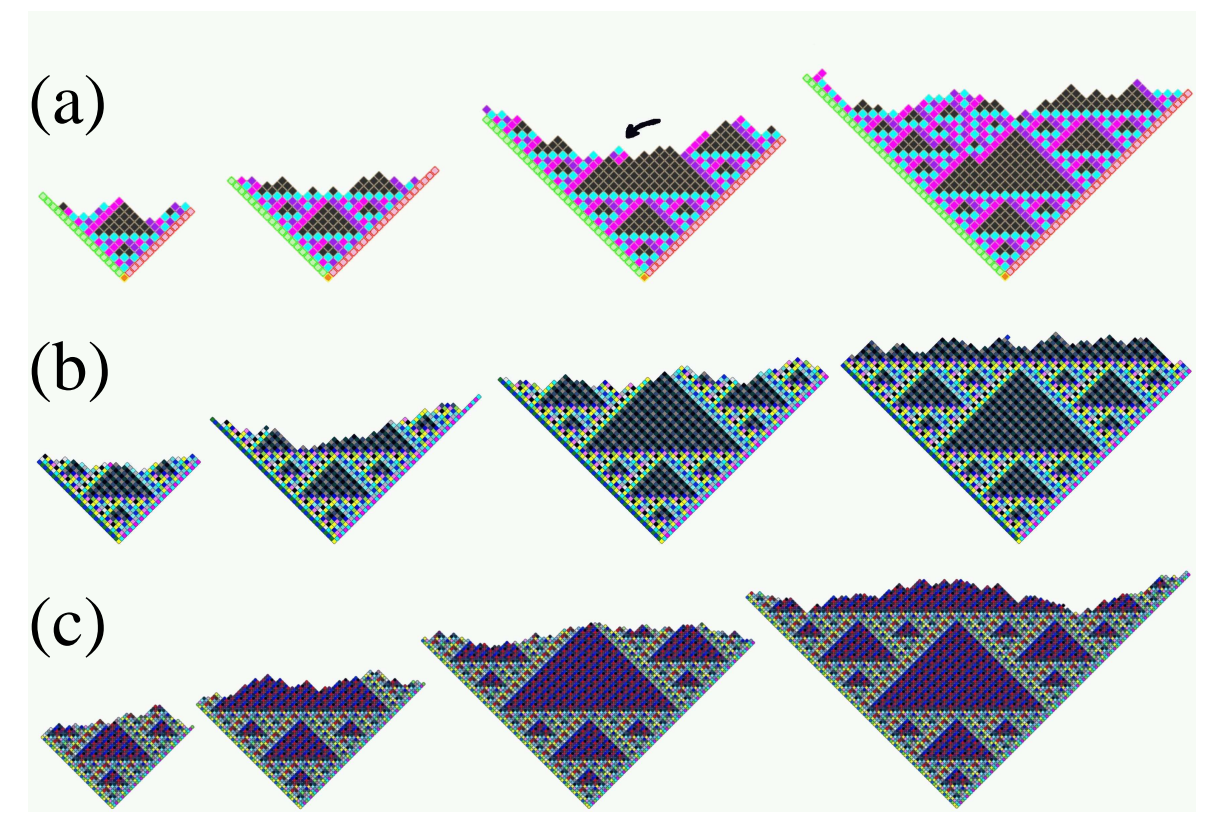

Fig. 4. (a) Growth of the original $(1 \times 1)$ Sierpinski tile set at $G_{m c}=13.9$ and $G_{s e}=$ 7.0 , to a size of $\sim 32$ layers in $\sim 530$ simulated seconds. Two errors can be seen; the first occurs in the third frame and is indicated by an arrow. Subsequent error-free growth correctly propagates the erroneous information. (b) Growth of the $2 \times 2$ proofreading tiles at $G_{m c}=12.9$ and $G_{s e}=6.5$, to a size of $\sim 64$ layers in $\sim 460$ simulated seconds. (c) Growth of the $3 \times 3$ proofreading tiles at $G_{m c}=11.9$ and $G_{s e}=6.0$, to a size of $\sim 96$ layers in $\sim 310$ simulated seconds.

if such a tile arrives before the mismatched tile dissociates, the mismatched tile becomes locked in by multiple bonds, and is now unlikely to dissociate. For a direct $\mathrm{BCA}$ tile set, the kinetic trap model shown in figure $3 \mathrm{~b}$ accurately predicts growth errors in kTAM simulations to obey [28]

$$
1-\varepsilon \approx \frac{1}{1+2 N \frac{r^{*}+r_{r, 2}}{r^{*}+r_{r, 1}}} \text { and thus } \varepsilon \approx 2 N \frac{r^{*}+r_{r, 2}}{r^{*}+r_{r, 1}} \underset{r^{*} \rightarrow 0}{\longrightarrow} 2 N e^{-G_{s e},}
$$

where $r=\frac{1}{2}\left(r_{f}-r_{r, 2}\right)$ is the overall growth rate and $r^{*}=\alpha r$ is the effective rate at which sites are frozen in the model. $\alpha=1.5$ is a free parameter chosen fit to the data; in some sense, it accounts for the fluctuations of the growth process, in which the growth front will wash back and forth over a given site several times before it is "frozen" in place.

The kinetic trapping theory identified a critical relationship. Although arbitrarily low error rates can be achieved by appropriate choice of $G_{m c}$ and $G_{s e}$, they come at the cost of a significant slow-down. This trade-off can be visualized by plotting $r$ vs $\varepsilon$ for all reasonable values of $G_{m c}$ and $G_{s e}$. As illustrated by the upper $(1 \times 1)$ plots in figure 5 , all points lie above $r \approx \beta \varepsilon^{2}$, where 
Simulation and Kinetic Trap Predictions for Proofreading Tiles

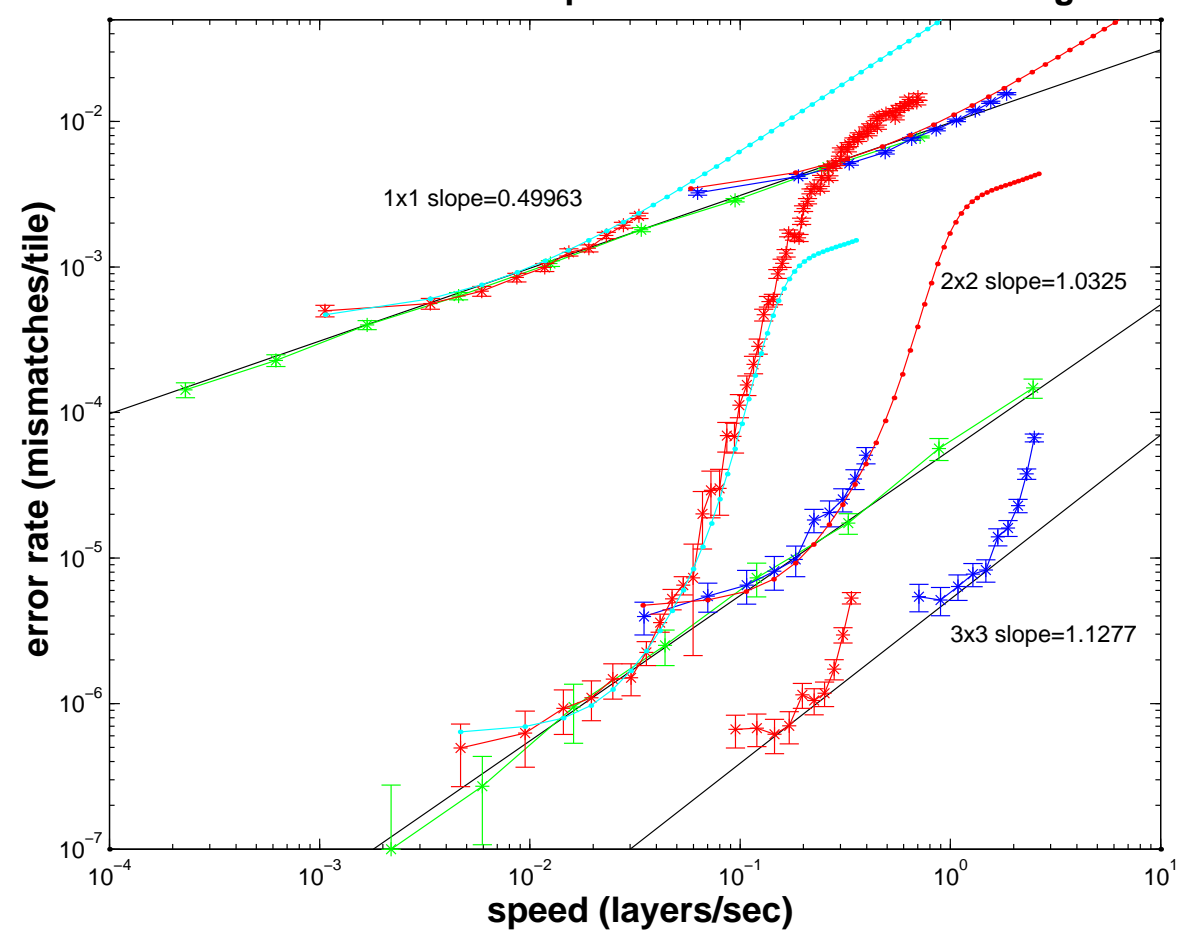

Fig. 5. Plot of error rate vs. growth speed as measured in simulations (*'s) and as according to kinetic trap theory (lines). Here, $G_{m c}=2 * G_{s e}-\epsilon$; i.e., $\epsilon$ measures how far the reaction is from the $\tau=2$ melting transition. Curved lines (red and blue) vary $G_{m c}$ and $\epsilon$ for fixed $G_{s e}$, thus demonstrating how the growth speed decreases too near the melting transition and the error rates increases too far below the melting transition. (Simulations used $G_{s e}=6.5$ and 8.5 for $1 \times 1,6.5$ and 7.5 for $2 \times 2$, and 5.5 and 6.5 for $3 \times 3$ tile sets.) Straight lines (green) vary $G_{s e}$ and $G_{m c}$ for fixed $\epsilon$, thus following the line just below the melting transition in phase space to obtain a Pareto-optimal speed/error trade-off. (Simulations used $k_{f}=10^{6} / \mathrm{M} / \mathrm{sec}$ and $\epsilon=.2, .1, .15$ for $1 \times 1$, $2 \times 2$, and $3 \times 3$ tile sets respectively. Error bars show two standard deviations, computed using $\sigma=\sqrt{\varepsilon} / \sqrt{m}$ where $m$ is the total number of tiles grown in all simulations using a given $G_{s e}$ and $G_{m c}$. A variable number of simulation runs were used, chosen to make the error bars small. )

$\beta \approx 1 \times 10^{4} / \mathrm{M} / \mathrm{sec}$ is determined by how far below the melting temperature gives the optimal trade-off. ${ }^{6}$ This defines the Pareto-optimal boundary along which $r$ is the fastest growth rate that achieves error rate $\varepsilon$. Thus, decreasing error rates by a factor of 10 entails slowing down the self-assembly process by a factor of 100 . This is bad; results on fault-tolerant computing in the digital

${ }^{6}$ If $G_{m c}=2 G_{s e}-\epsilon$, then $r=\frac{1}{2}\left(r_{f}-r_{r, 2}\right)=\frac{1}{2} k_{f}\left(e^{\epsilon}-1\right) e^{-2 G_{s e}} \approx \frac{k_{f}}{8 N^{2}} \varepsilon^{2}$ for equilibrium error rates. This estimate is accurate only within an order of magnitude. 
circuit model typically entail a logarithmic, rather than quadratic, slow-down $[24,15]$.

In the rest of the paper, we give a construction of "proofreading" tile sets to implement arbitrary BCA, in which each original tile is replaced by a $K \times K$ block of tiles in the new tile set. ${ }^{7}$ Simulation and theory results for $2 \times 2$ and $3 \times 3$ tile sets are shown in figures 4 and 5 , showing scaling behavior of roughly $r \approx \beta \varepsilon^{1.0}$ and $r \approx \beta \varepsilon^{0.89}$ respectively, with $\beta$ varying by less than a factor of five depending on the tile set. This is a significant improvement: for target error rates of $10^{-4}$, the $2 \times 2$ proofreading tiles result in a $10^{4}$-fold speed-up over the original tile set. Similarly, for the same physical conditions that result in a $1 \%$ error rate for the original tile set, the $2 \times 2$ proofreading tile set yields a $0.01 \%$ error rate, and the $3 \times 3$ proofreading tile set yields a $0.001 \%$ error rate. This is extremely encouraging for experimental studies of algorithmic self-assembly: conditions in which perfect $10 \times 10$ Sierpinski triangles can now be grown may yield $100 \times 100$ triangles with proofreading tiles. Still, these constructions result in greater slow-down than achieved in the digital circuit model, suggesting that further improvements await discovery.

The remainder of this paper describes the proofreading tile set construction, gives an explanation of the principles by which it works, and comments on its limitations.

\section{$3 \quad$ Proofreading tile sets}

The ability for the kTAM to discriminate between tiles that partially or perfectly match a growth site relies on the cooperativity of sticky-end binding: the binding of one sticky end stabilizes the binding of the other sticky end. The basic idea of proofreading tiles is to exploit cooperative binding at the next higher level: to have several tiles that stabilize each other when they bind together. Cooperative binding is a common feature of transcription factors in genetic regulatory networks [16] and has been examined as a mechanism for increased sensitivity in one-dimensional self-assembly processes $[2,3]$. New issues arise in the context of two-dimensional self-assembly.

The general $2 \times 2$ proofreading construction is shown in figure $6 \mathrm{a}$, and its application to the Sierpinski tile set is shown in figure 6bcd. Essentially, each rule tile in the original tile set is replaced by four tiles with related labels. Arranged in a $2 \times 2$ block, the sides of the block present the same logical labels as the original tile. The side internal to the block are given unique labels, not shared by tiles from any other block. Thus, assembly from the seed tile according to the aTAM proceeds according to the same logic as the original tile set, but scaled up in size by a factor of two. ${ }^{8}$ However, in the kTAM, a new phenomenon can be observed when a mismatched tile is incorporated: there is now no way to continue

\footnotetext{
7 The direct BCA tile sets can be considered to be the $1 \times 1$ proofreading construction.

${ }^{8}$ For self-similar patterns like the Sierpinski triangle, the resolution of the resulting pattern remains the same - each $2 \times 2$ block can be labeled according to the precomputed pattern.
} 
(a)

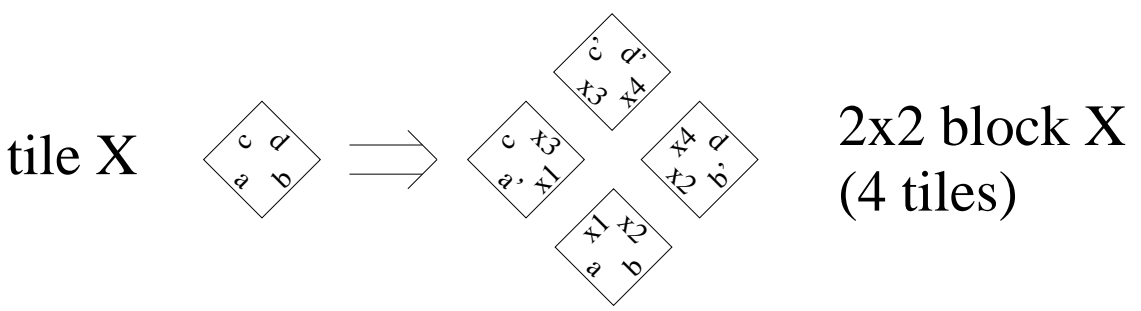

rule tiles

(b)

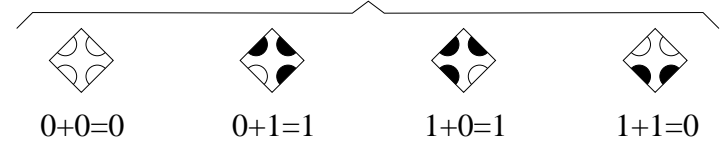

boundary tiles

(c)

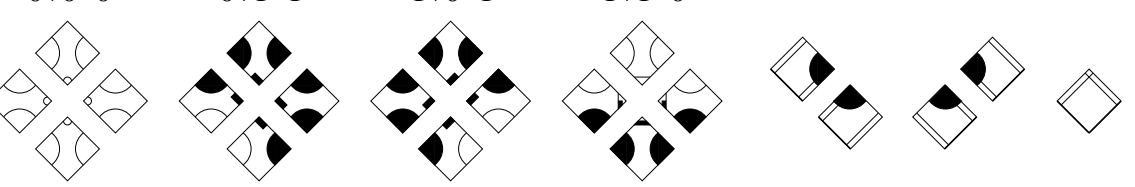

(d)

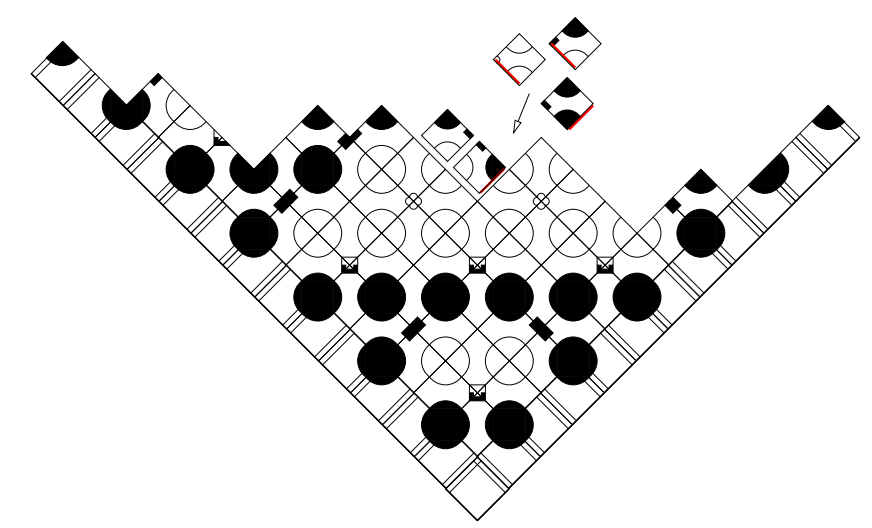

Fig. 6. (a) The general $2 \times 2$ proofreading construction for rule tiles. (b) The original Sierpinski tiles. (c) The $2 \times 2$ proofreading Sierpinski tiles. (d) Growth of the proofreading Sierpinski tiles. Small tiles illustrate that when a mismatched tile is incorporated, further growth on one side must involve a second mismatch.

growth without making an additional error. This is illustrated by the small tiles in figure 6d: after the initial (lowest) small tile arrives, forming a mismatch on one side, any further tile assembling on that side will either (a) agree with the initial tile but, because it therefore must be part of the same proofreading block, mismatch on its lower right side, or (b) agree with its lower right input, but therefore form a mismatch with the initial small tile. The assembly process stalls, giving time for the initial mismatched tile to fall off and be replaced by a correct tile. The final assembly therefore has no record of the mishap having occurred. 
(a)

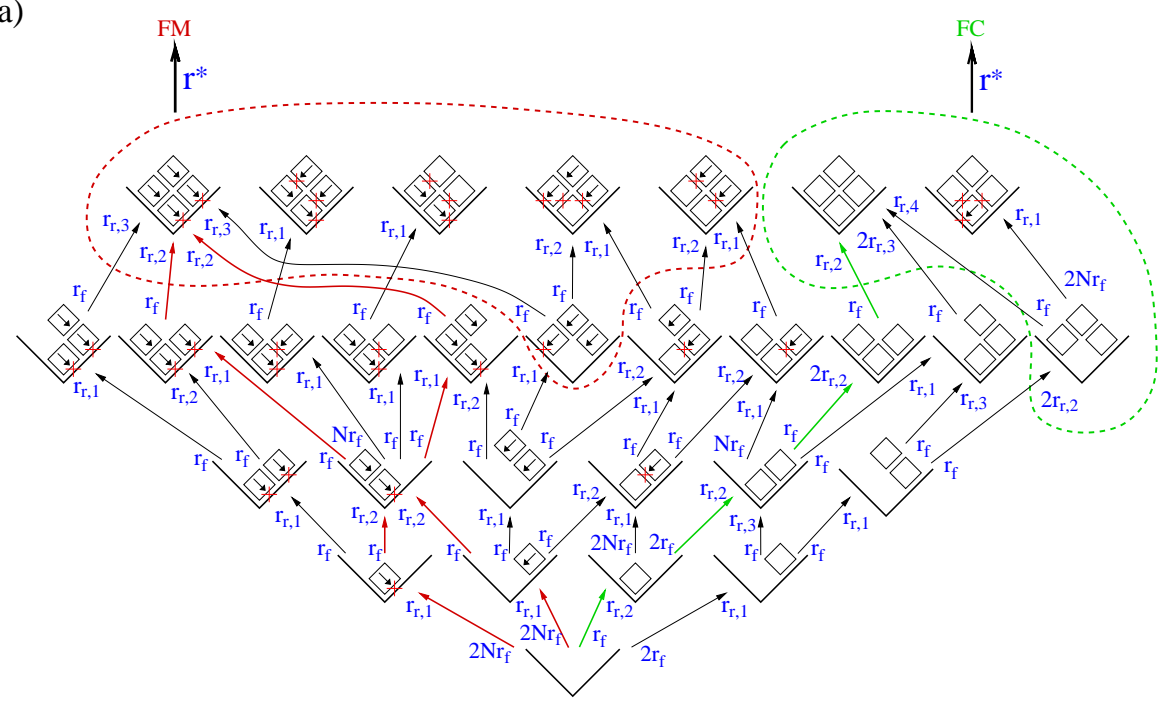

(b)

$$
\mathrm{FC} \rightleftharpoons \mathrm{r}_{4} \underset{2 \mathrm{r}_{\mathrm{r}, 2}}{\stackrel{\mathrm{r}_{\mathrm{f}}}{\rightleftarrows}} \mathrm{M}_{3} \underset{\mathrm{r}_{\mathrm{r}, 1}}{\stackrel{2 \mathrm{r}_{\mathrm{f}}}{\rightleftharpoons}} \mathrm{M}_{2} \underset{2 \mathrm{r}_{\mathrm{r}, 2}}{\stackrel{\mathrm{r}_{\mathrm{f}}}{\rightleftharpoons}} \mathrm{M}_{1} \stackrel{4 \mathrm{Nr}_{\mathrm{f}}}{\underset{\mathrm{r}_{\mathrm{r}, 1}}{\rightleftharpoons}} \mathrm{E} \underset{\mathrm{r}_{\mathrm{r}, 2}}{\stackrel{\mathrm{r}_{\mathrm{f}}}{\rightleftharpoons}} \mathrm{C}_{1} \underset{\mathrm{r}_{\mathrm{r}, 2}}{\stackrel{2 \mathrm{r}_{\mathrm{f}}}{\rightleftharpoons}} \mathrm{C}_{2} \underset{2 \mathrm{r}_{\mathrm{r}, 2}}{\stackrel{\mathrm{r}_{\mathrm{f}}}{\rightleftharpoons}} \mathrm{C}_{3} \underset{\mathrm{r}_{\mathrm{r}, 2}}{\stackrel{\mathrm{r}_{\mathrm{f}}}{\rightleftharpoons}} \mathrm{C}_{4} \stackrel{\mathrm{r}^{*}}{\longrightarrow} \mathrm{FC}
$$

Fig. 7. (a) A kinetic trapping model including all 29 states (up to symmetry) representing "well-associated" tiles within a $2 \times 2$ growth site (see text for details). Arrows inside tiles indicate that the tile belongs to a proofreading block that has a mismatch to the input in the indicated direction; there are $N$ such tiles for an $(N+1)$-state $\mathrm{BCA}$. Arrows between states indicate reversible reactions (association or dissociation of a tile); reverse reaction rates are given at the head of each arrow, and forward reaction rates are given near the tail. States within dotted circles each have an irreversible reaction to a frozen state (either FM or FC) with rate $r^{*}$. (b) A simplified kinetic trapping model with 9 states considers only the major reaction pathways in (a), which are indicated by the red and green reaction arrows for pathways leading to mismatched or correct blocks, respectively.

The forcing of errors to be co-localized in pairs results in the error rate being squared relative to the original tile set. A detailed kinetic trapping model for $2 \times 2$ proofreading tiles (shown in figure $7 \mathrm{a}$ ) produces excellent agreement ${ }^{9}$ with the simulation results (shown in figure 5), including the precipitous decline in reliability as physical conditions move away from the melting transition. Each state in this model represents a possible arrangement of tiles (up to symmetries) within the $2 \times 2$ growth site. Each tile could be either part of the correct block for that site (unlabeled) or part of a block that has a mismatch on one side or the other (indicated by the direction of the arrow; there are $N$ such blocks

\footnotetext{
9 Again, the value of $r^{*}$ was determined by fitting free parameters to best match the data. Here, we used $r^{*}=\alpha r e^{\gamma \epsilon}$ where $\epsilon=2 G_{s e}-G_{m c}, \alpha=1$, and $\gamma=12$. We have no strong justification for this formula.
} 
for BCA tile sets). The 29 states considered are all those in which the tiles are "well-associated", that is, we prune the full model by removing all states in which more than one tile is attached by only one bond or in which some tile is attached by no bonds at all - such states would be very short-lived.

A simplified model (shown in figure $7 \mathrm{~b}$ ) considers just the dominant pathways (red and green reaction arrows in figure 7a), and yields similar results near the melting transition. The improvement in the error rate near the melting transition, then, is due to the elementary features preserved in this model, namely that (a) the correct block can be formed via a series of four favorable steps, and (b) every path to a mismatch contains at least two significantly unfavorable steps with a fast $r_{r, 1}$ reverse reaction.

Although baroque, both models can easily be solved numerically by representing the transition rates in matrix form and computing the steady-state by matrix inverse.

The basic phenomenon can be more easily understood using the following intuition. Consider first the direct BCA tile set. Optimal growth rates are obtained near the melting temperature of the crystals, where $G_{m c} \approx 2 G_{s e}$. Under these conditions, the error rate reaches the thermodynamic limit of $\varepsilon \approx e^{-\Delta G / R T}=$ $e^{-G_{s e}}$, where $\Delta G$ is the difference in free energy between an assembly with a mismatched tile and one with a correct tile. However, the growth rate will be proportional to the monomer tile concentration, $r \approx \beta[D X]=\beta e^{G_{m c}}$. This yields the scaling relation for the original tile set, $r \approx \beta \varepsilon^{2}$.

This type of argument can be generalized for $K \times K$ proofreading constructions, wherein each tile is replaced by a $K \times K$ block of unique tiles. Optimal growth rates still occur near the melting temperature $\left(G_{m c} \approx 2 G_{s e}\right)$, and the growth rate is still $r \approx \beta[D X]=\beta e^{G_{m c}}$. However, the thermodynamic error rate (for an entire block) is now determined by the minimal error, which involves at least $K$ mismatched tiles. ${ }^{10}$ Thus $^{11} \varepsilon \approx e^{-\Delta G / R T}=e^{-K G_{s e}}$, and the argument yields $r \approx \beta \varepsilon^{2 / K}$.

If the above reasoning held for all $K$, and the proofreading block size were to be chosen based on the target error rate needed, a logarithmic dependence of $K$ on the target error rate would be achieved for constant physical conditions. This matches what has been found for digital circuits. Unfortunately, although the argument correctly predicts the scaling behavior for $K=2$, the simulation results for $K=3$ (figure 5 ) and $K=4$ (data not shown) fall short of the prediction.

We attribute this failure to a second mechanism for growth errors. Whereas proofreading tiles perform well at correcting errors during growth at a site where

$\overline{10}$ Since each erroneous block involves $K^{2}$ tiles and typically contains exactly $K$ mismatches, the per-block error rates $\varepsilon_{\text {block }}=K \varepsilon$, where $\varepsilon$ is the per-tile error fraction. (Tile errors are now clearly not iid.) We found it more convenient to report simulation results in terms of the per-tile error rate.

11 This argument illustrates why it is necessary to use cooperativity by relying on the independent assembly of the $K^{2}$ pieces in a block. If one were to use a preassembled block, the melting temperature would occur at $G_{m c} \approx 2 K G_{s e}$, giving rise to the original $r \approx \beta \varepsilon^{2}$ scaling behavior. 
correct growth could occur, they do nothing to prevent errors due to spontaneous growth on a facet ("roughening"), as shown in figure 8a. Ensuring that boundaries grow at approximately the same speed as the interior ${ }^{12}$ reduces the amount of faceting, but even so, facets of length $n$ appear with a frequency of $\approx 2^{-n}$, making facet growth errors rare but unavoidable. Some other error-correcting strategy will be necessary to prevent this type of error.

\section{Strong bonds, capping tiles, and self-repair}

It is important to realize that the proofreading tile set construction given here doesn't work for all tile sets. The existence of the alternative growth error mechanism on facets implies that tile sets whose growth process intrinsically involves facets will fail to derive great benefit from proofreading tiles. For example, the the tile set presented in [20] for growing $M \times M$ squares using $O(\log M)$ tiles results in a final assembly in which three sides are facets - i.e., each tile on those sides displays a strength- 1 bond, but under $\tau=2$ aTAM rules, no growth can occur. In the kTAM, growth will eventually occur, ruining the desired structure, as shown in figure $8 \mathrm{~b}($ top) for a $26 \times 26$ square. Proofreading tiles do nothing to fix this problem.

To reliably construct an $M \times M$ square required modifying the original tile set in three ways: first, identifying the direction of growth and specializing the tiles so that each tile is used for only one direction of growth, and each sticky end appears on as few tiles as possible; second, making use of additional "capping" tiles to quickly cover the final facets of the square with tiles that have null bonds on their outer sides, thus reducing errors due to roughening; and third, using two perpendicular binary counters to encourage the growth front to avoid large facets. Assembly using this improved tile set is shown in figure $8 \mathrm{~b}$ (middle) for a $49 \times 49$ square.

With facet roughening errors ameliorated, it makes sense to ask whether now proofreading further decreases the error rate. However, because growth of the square within the counter region takes a meandering path making use of several rule tiles with strength- 2 bonds, the proofreading construction must be extended to blocks involving strength- 2 bonds. This construction, shown in figure $8 \mathrm{c}$, can only be used for tile sets in which each tile occurs only during growth in a particular direction; i.e., its input and output sides can be determined and are used consistently. The bond labels on proofreading tiles are as described previously, but the strength of those bonds is determined by the growth direction (for the input sides) or by the growth direction of subsequent tiles (for the output sides). This $2 \times 2$ proofreading construction was applied to the improved tile set described above, with results shown in figure $8 \mathrm{~b}$ (bottom).

Out of 31 trials, the original tile set never formed the desired $26 \times 26$ squares properly, the improved tile set was $65 \%$ successful at forming $49 \times 49$ squares,

12 This is the case in the simulations, thanks to the boundary tile concentration being set to $\frac{1}{2}$ the concentration of the rule tiles. 

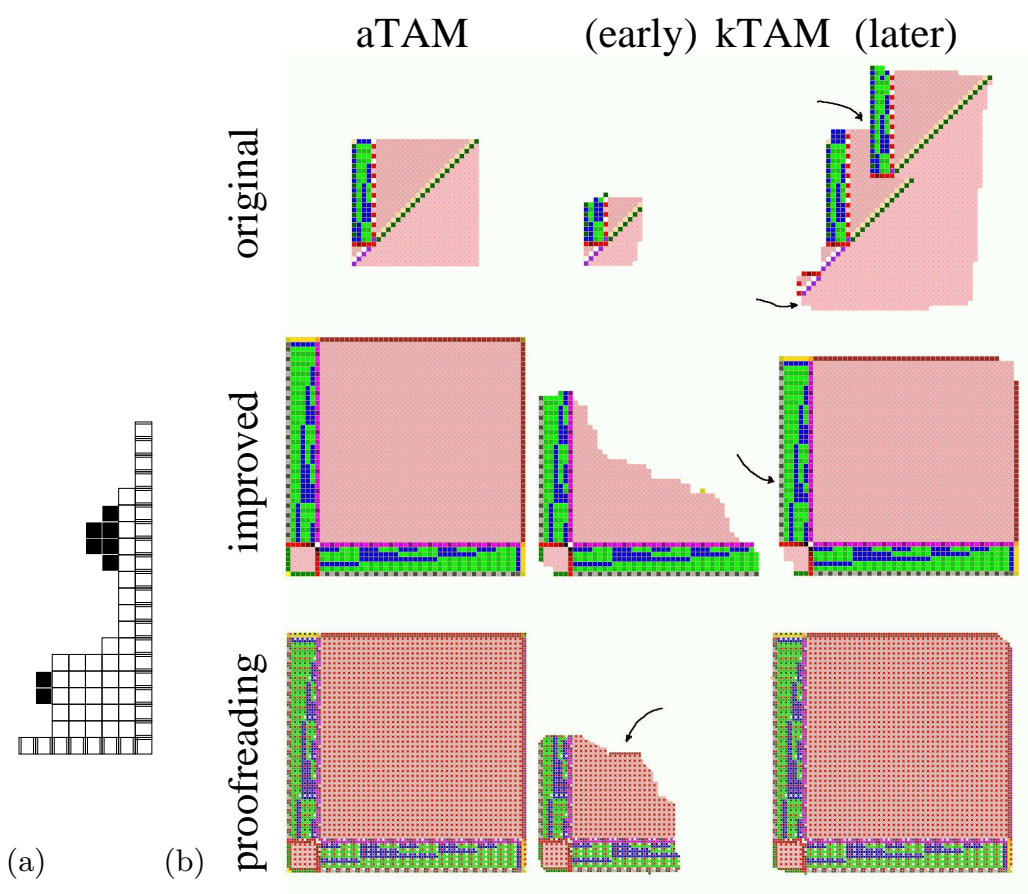

(c)
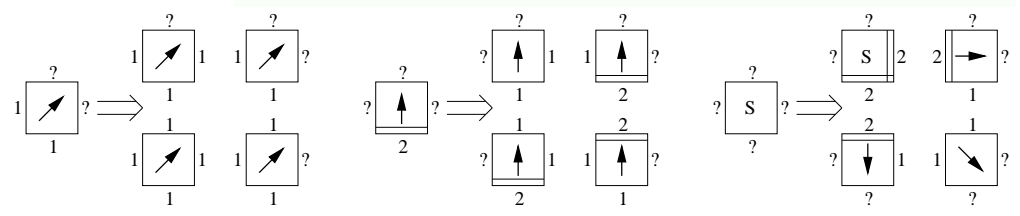

Fig. 8. (a) Small black tiles illustrate spurious nucleation of the subsequent layer (facet roughening). The information content of these tiles is likely to be wrong. Our proofreading tile set construction does not efficiently correct these errors. (b) From left to right: Assembly according to the aTAM at $\tau=2$; early growth in the kTAM at $G_{m c}=17.7$ and $G_{s e}=9.0$; and the resulting structure. From top to bottom: The binary counter construction for assembling $M \times M$ squares; the improved twocounter construction with capping tiles; and the $2 \times 2$ proofreading construction for the latter. All tile sets assemble perfectly under the aTAM. Under the kTAM, the one-counter tile set fails even for a small square because spurious nucleation on facets frequently results in uncontrolled growth (arrows). The two-counter tile set still suffers from algorithmic errors (arrow), but the capping tiles significantly reduce spurious nucleation of new layers. The proofreading construction reduces these problems only somewhat for this tile set. The arrow indicates a facet roughening error in which capping tiles prematurely nucleated on the growth front. In this run, these capping tiles are eventually displaced by the growth of correct tiles. (c) The scheme for assigning bond strengths for proofreading tiles when the tile set contains strength- 2 bonds. Arrows indicate the direction of growth for correct use of the tile, and " $\mathrm{S}$ " indicates the seed tile. The strength of bonds on sides marked by "?" is dictated by the tiles that bind to them in a correct assembly; if no tiles bind to them, strength-0 bonds are used. 


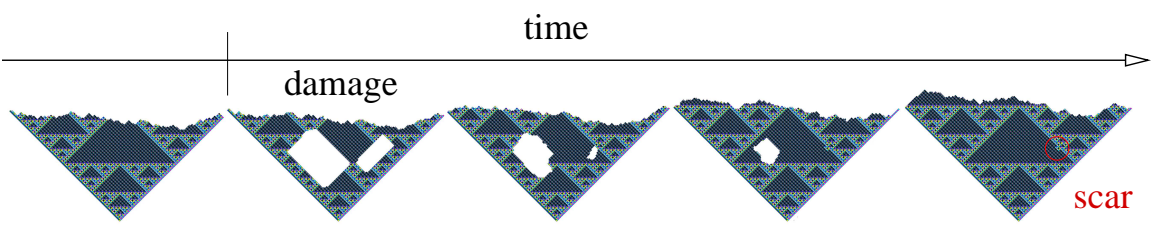

Fig. 9. Proofreading tile sets are often able to heal a puncture in the crystal. Sometimes, as in this case, some of the tiles that fill in the puncture do not perfectly match their neighbors - a form of "scar tissue."

and the proofreading tile set formed $98 \times 98$ squares $87 \%$ of the time. For comparison, the improved tile set formed $99 \times 99$ squares only $26 \%$ of the time. The disappointingly modest ${ }^{13}$ benefit provided by the proofreading tiles suggests that alternative error mechanisms are dominant for these tile sets.

The proofreading tiles also give rise to some surprising, and pleasant, behaviors. One such phenomenon is their ability to heal punctures of the growing crystal, as shown in figure 9. Since the identity of tiles within the punctured region is uniquely determined by the perimeter of that region (in fact, just the lower portion of the perimeter suffices), one might expect even the non-proofreading tile set to be able to correctly fill in the punctured region. However, regrowth into the punctured region may occur in any direction, including backward from the most advanced edge of the puncture, where there are multiple ways to proceed locally when using tiles implementing irreversible BCA, such as the Sierpinski tiles. ${ }^{14}$ Thus, regrowth is not always perfect; indeed, the direct BCA tiles very often leave "scar tissue", although the proofreading tiles do so much less frequently.

\section{Discussion}

The primary result of this paper is that dramatic improvements in the error rates for algorithmic self-assembly can be achieved, in principle, by proper redesign of the abstract tile set, without changing the fundamental molecular implementation. The $2 \times 2$ proofreading tiles for BCA increase the optimal growth speed $r$ for which a target error rate $\epsilon$ can be achieved from $r=\beta \epsilon^{2}$ to $r=\beta \epsilon$, and greater improvements can be obtained with larger $K \times K$ proofreading constructions. If these constructions hold up in practice, it may be possible for algorithmic self-assembly to scale up to macroscopic assemblies without errors.

Although the theoretical and simulation results presented here firmly establish the effectiveness of the proofreading tile construction, the rigorous proof of their robustness in the kTAM remains an open problem.

When considering implementation of proofreading tile sets with DNA tiles, the question of efficiency arises. One wishes for a minimal number of tiles, since

${ }^{13}$ The $2 \times 2$ proofreading Sierpinski tile set under these conditions would obtain $\varepsilon=$ $1.5 \times 10^{-7}$, and therefore $10^{4}$ tiles would assemble without errors $99.9 \%$ of the time.

${ }^{14}$ As pointed out by Adleman (personal communication), this suggests a relationship between reversible cellular automaton logic and self-healing properties. 
(a)

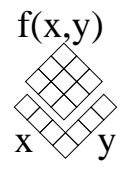

(b)

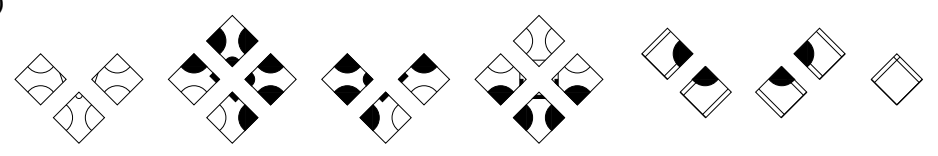

Fig. 10. (a) Schema for the optimized tile set, in which every pair of inputs $x, y$ is given unique tiles for the edge of a $K \times K$ block, and the remaining $(K-1) \times(K-1)$ tiles is shared for each output $f(x, y)$. (b) An optimized $2 \times 2$ proofreading tile set for the Sierpinski tiles.

each additional tile requires a significant amount of laboratory work. Fortunately, for tile sets implementing BCA for which both outputs are identical (i.e. $f_{1}=f_{2}=f$, as is the case for the Sierpinski tiles), blocks that output the same value $f(x, y)$ can share the same $(K-1) \times(K-1)$ sub-block, as shown in figure 10, achieving approximately the same fault-tolerance while using only $(2 K-1) N^{2}+(K-1)^{2} N$ instead of $K^{2} N^{2}$ rule tiles. For DNA tiles made from DAO-E molecules, for which two DNA tile must be created for each abstract tile (subject to exploitation of symmetries) [21], $2 \times 2$ proofreading increases the number of DNA rule tiles from 6 for the original Sierpinski tile set to 9 for proofreading - a very moderate increase. We are therefore exploring this tile set experimentally.

Other optimizations are possible, such as trying to reduce the increase in scale of the final pattern produced by self-assembly. Reif [17] has already proposed constructions that improve upon the $K$-fold spatial scaling inherent here.

Furthermore, it is at present unclear how these constructions can be adapted for general tile sets, where the growth front may involve significant faceting. It appears that an additional proofreading mechanism will be required to correct for errors that occur due to premature growth on a facet; the concept of "invadable tiles" may help here [6].

Robustness to assembly errors may be contrasted with robustness to implementation errors, wherein the molecular tiles fail to conform to the desired specifications, as expressed within the kTAM. For example, bond strengths for different labels that should all be identical (e.g., weak) may vary somewhat in a specific set of DNA tiles. Similarly, an experiment may provide the tiles at slightly different (rather than identical) concentrations. More seriously, as assembly proceeds in an undisturbed reaction, monomer tile concentrations will be depleted over time. Also, monomer tiles may aggregate into small assemblies, rather than accreting one-by-one onto the growing crystal. It is hoped that proofreading tiles, in addition to providing robustness to assembly errors, will provide improved robustness to implementation errors, but this issue has not yet been investigated.

Finally, our experience with experimental systems suggests that a major remaining issue is constraining assembly to begin only from the selected seed tile, i.e., minimizing spontaneous spurious nucleation of rule tiles or boundary tiles. Careful exploitation of critical nucleus size during supersaturation appears to provide a solution to this quandary [22]. 


\section{Acknowledgments}

This work benefited from discussions with Leonard Adleman, Matthew Cook, Ashish Goel, Paul Rothemund, Rebecca Schulman, Georg Seelig, David Soloveichik, and Chris Umans. Thanks to John Reif for encouraging me to write this up and for sharing his unpublished manuscript. EW and RB were supported by NSF CAREER Grant No. 0093486, DARPA BioComputation Contract F30602-01-20561, NASA NRA2-37143, and GenTel. Simulation code and tile sets used in this paper, as well as MATLAB scripts for evaluating the kinetic trapping models, may be obtained from http://www. dna.caltech.edu/SupplementaryMaterial.

\section{References}

1. L. M. Adleman. Molecular computation of solutions to combinatorial problems. Science, 266:1021-1024, Nov. 11, 1994.

2. R. Bar-Ziv and A. Libchaber. Effects of DNA sequence and structure on binding of RecA to single-stranded DNA. Proc. Nat. Acad. Sci. USA, 98(16):9068-9073, 2001.

3. R. Bar-Ziv, T. Tlusty, and A. Libchaber. Protein-DNA computation by stochastic assembly cascade. Proc. Nat. Acad. Sci. USA, 99(18):11589-11592, 2002.

4. V. A. Bloomfield, D. M. Crothers, and I. Tinoco, Jr. Nucleic Acids: Structures, Properties, and Functions. University Science Books, 2000.

5. B. A. Bondarenko. Generalized Pascal Triangles and Pyramids, Their Fractals, Graphs and Applications. The Fibonacci Association, 1993. Translated from the Russion and edited by Richard C. Bollinger.

6. H.-L. Chen, Q. Cheng, A. Goel, M. deh Huang, and P. M. de Espanés. Invadable self-assembly: Combining robustness with efficiency. ACM-SIAM Symposium on Discrete Algorithms (SODA), to appear, 2004.

7. J. Chen and J. Reif, editors. DNA Computing 9, Berlin Heidelberg, to appear. Springer-Verlag.

8. M. Cook, P. W. K. Rothemund, and E. Winfree. Self-assembled circuit patterns. In Chen and Reif [7].

9. T.-J. Fu and N. C. Seeman. DNA double-crossover molecules. Biochemistry, 32:3211-3220, 1993.

10. P. Gács. Reliable cellular automata with self-organization. Journal of Statistical Physics, 103(1/2):45-267, 2001.

11. T. H. LaBean, H. Yan, J. Kopatsch, F. Liu, E. Winfree, J. H. Reif, and N. C. Seeman. Construction, analysis, ligation, and self-assembly of DNA triple crossover complexes. Journal of the American Chemical Society, 122:1848-1860, 2000.

12. M. G. Lagoudakis and T. H. LaBean. 2-D DNA self-assembly for satisfiability. In E. Winfree and D. K. Gifford, editors, DNA Based Computers V, volume 54 of DIMACS, pages 141-154, Providence, RI, 2000. American Mathematical Society.

13. C. Mao, T. H. LaBean, J. H. Reif, and N. C. Seeman. Logical computation using algorithmic self-assembly of DNA triple-crossover molecules. Nature, 407(6803):493496, 2000.

14. C. Mao, W. Sun, and N. C. Seeman. Designed two-dimensional DNA Holliday junction arrays visualized by atomic force microscopy. Journal of the American Chemical Society, 121(23):5437-5443, 1999. 
15. N. Pippenger. Developments in "the synthesis of reliable organisms from unreliable components". In The Legacy of John von Neumann, pages 311-324. American Mathematical Society, 1990.

16. M. Ptashne. A Genetic Switch, 2nd ed. Cell Press \& Blackwell, 1992.

17. J. Reif. Compact error-resilient computational DNA tiling assemblies. Unpublished manuscript.

18. J. Reif. Local parallel biomolecular computing. In H. Rubin and D. H. Wood, editors, DNA Based Computers III, volume 48 of DIMACS, pages 217-254, Providence, RI, 1999. American Mathematical Society.

19. P. W. K. Rothemund and E. Winfree. Algorithmic self-assembly of DNA Sierpinski triangles. In preparation.

20. P. W. K. Rothemund and E. Winfree. The program-size complexity of selfassembled squares. In Symposium on Theory of Computing (STOC). ACM, 2000.

21. R. Schulman, S. Lee, N. Papadakis, and E. Winfree. One dimensional boundaries for DNA tile assembly. In Chen and Reif [7].

22. R. Schulman and E. Winfree. Controlling nucleation rates in algorithmic selfassembly. In preparation.

23. D. Soloveichik and E. Winfree. Complexity of self-assembled scale-invariant shapes. Submitted.

24. J. von Neumann. Probabilistic logics and the synthesis of reliable organisms from unreliable components. In C. E. Shannon and J. McCarthy, editors, Automata Studies, pages 43-98. Princeton University Press, 1956.

25. H. Wang. Proving theorems by pattern recognition. II. Bell System Technical Journal, 40:1-42, 1961.

26. H. Wang. Dominoes and the AEA case of the decision problem. In J. Fox, editor, Proceedings of the Symposium on the Mathematical Theory of Automata, pages 23-55, Brooklyn, New York, 1963. Polytechnic Press.

27. E. Winfree. On the computational power of DNA annealing and ligation. In R. J. Lipton and E. B. Baum, editors, DNA Based Computers, volume 27 of DIMACS, pages 199-221, Providence, RI, 1996. American Mathematical Society.

28. E. Winfree. Simulations of computing by self-assembly. Technical Report CSTR:1998.22, Caltech, 1998.

29. E. Winfree, F. Liu, L. A. Wenzler, and N. C. Seeman. Design and self-assembly of two-dimensional DNA crystals. Nature, 394:539-544, 1998.

30. E. Winfree, X. Yang, and N. C. Seeman. Universal computation via self-assembly of DNA: Some theory and experiments. In L. F. Landweber and E. B. Baum, editors, DNA Based Computers II, volume 44 of DIMACS, pages 191-213, Providence, RI, 1998. American Mathematical Society.

31. H. Yan, S. H. Park, G. Finkelstein, J. H. Reif, and T. H. LaBean. DNA-templated self-assembly of protein arrays and highly conductive nanowires. Science, 301:18821884, 2003. 